\title{
Calidad de vida del paciente con epilepsia y sobrecarga de su cuidador
}

\section{Quality of life in patients with epilepsy and caregivers' burden}

\author{
Arturo García-Galicia', Luis A. Toral-Gámez², Omar A. Martínez-Fernández³, Álvaro J. Montiel-Jarquín*, \\ Akihiki M. González-López ${ }^{5}$ y José Á. Parra-Salazar ${ }^{4}$ \\ ${ }^{1}$ Unidad Médica de Alta Especialidad, Hospital de Traumatología y Ortopedia, Centro Médico Nacional General Manuel Ávila Camacho, IMSS, \\ Puebla; ${ }^{2}$ Unidad Médica de Alta Especialidad, Hospital de Pediatría, Centro Médico Nacional de Occidente, IMSS, Jalisco, ${ }^{3}$ Unidad Médica de \\ Alta Especialidad, Hospital General Dr. Gaudencio González Garza, Centro Médico Nacional La Raza, IMSS, Ciudad de México; ${ }^{4}$ Unidad Médica \\ de Alta Especialidad, Hospital de Especialidades de Puebla, Centro Médico Nacional Gral. Manuel Ávila Camacho, IMSS, Puebla; ${ }^{5}$ Unidad de \\ Medicina Familiar n. ${ }^{\circ}$, Delegación Puebla, IMSS, Puebla. México
}

\begin{abstract}
Resumen
Introducción: La epilepsia es una enfermedad que afecta la calidad de vida. La padecen 1.4 millones de pacientes menores de 15 años en todo el mundo. La calidad de vida relacionada con la salud (CVRS) mide el impacto en la vida del paciente y su salud. Las principales afectaciones de los niños con epilepsia son la disminución de la cognición y el fracaso escolar. Objetivo: Determinar la CVRS del niño con epilepsia y la sobrecarga de su cuidador en un hospital de segundo nivel en Puebla, México. Pacientes y métodos: Estudio descriptivo, transversal, prospectivo, en niños de 4 a 10 años con epilepsia y en sus cuidadores primarios. Se incluyó a niños con diagnóstico de epilepsia y sus cuidadores, atendidos en consulta externa, con tratamiento de seis meses o más y de menos de cinco años. No se incluyó a pacientes con patologías concomitantes independientes de epilepsia que per se limitaran su calidad de vida. Se eliminó a aquellos que no completaron las escalas. Se aplicaron la escala de calidad de vida para pacientes epilépticos pediátricos y la escala de sobrecarga del cuidador de Zarit. Se empleó estadística descriptiva, $X^{2}$ y coeficiente de correlación de Spearman. Resultados: Se reclutó a 71 pacientes, 55\% niños y 45\% niñas, con una edad promedio de 6.7 años. La CVRS fue buena en el $76 \%$ y regular en el $24 \%$. Los dominios más afectados fueron la cognición y el comportamiento. La sobrecarga del cuidador primario fue poca-nula en el 53\% y leve-moderada en el 37\%, correlación con CVRS - 0.360 ( $p=0.002)$. Conclusiones: La correlación entre la CVRS y la sobrecarga de los cuidadores fue de débil a moderada. El control de las crisis epilépticas fue bueno, lo que disminuye la sobrecarga del cuidador.
\end{abstract}

Palabras clave: Calidad de vida. Epilepsia en niños. ECAVIPEP. Escala de Zarit. Sobrecarga del cuidador.

\section{Abstract}

Background: Epilepsy disease affects quality of life. There is 1.4 million under 15 years-old children affected worldwide. Impact in life patient and his health is assessed by Health-Related Quality of Life (HRQoL). Principal affectations are: cognitive handicap and scholar failure. Objective: To assess HRQoL in children with epilepsy and caregiver burden at a $2^{\text {nd }}$ level

Correspondencia:

*Álvaro J. Montiel-Jarquín

E-mail: dralmoja@ hotmail.com
Fecha de recepción: 06-02-2020

Fecha de aceptación: 03-03-2020

DOI: 10.24875/RHJM.20000023
Disponible en internet: 19-06-2020 Rev Hosp Jua Mex. 2020;87(2):81-87

www.revistahospitaljuarez.com 1405-9622/C 2020 Sociedad Médico-Quirúrgica del Hospital Juárez de México, A.C. Publicado por Permanyer. Este es un artículo open access bajo la licencia CC BY-NC-ND (http://creativecommons.org/licenses/by-nc-nd/4.0/). 
attention unit in Puebla, Mexico. Patients and methods: Descriptive, cross-sectional, prospective study, performed in 4-10 years-old children and their primary caregivers. Ambulatory children with epilepsy diagnosis and their caregivers were included, with six months-or more and less than five years treatment. Patients with pathologies different of epilepsy affecting $H R Q o L$ were excluded. Those (or their caregivers) who didn't complete scales were eliminated. ECAVIPEP and Zarit scales were applied. Descriptive stadistics, $X^{2}$ and Spearman coefficient were used. Results: 71 patients were recruited, 55\% boys and $45 \%$ girls, medium age 6.7 years. HRQoL was good in $76 \%$ and regular $24 \%$; most affected domains: cognition and behavior. Primary caregiver burden: few-none 53\%, low-moderated 37\%. Correlation HRQoL/Caregiver burden was -0.360 $(p=0.002)$. Conclusion: Correlation between HRQoL and Caregiver burden was weak. Control of epileptic seizures was good, with their caregiver's burden decreased.

Key words: HRQoL. Epilepsy in children. ECAVIPEP. Zarit Scale. Caregiver burden.

\section{Introducción}

La epilepsia es una enfermedad crónica que se manifiesta por descargas eléctricas cerebrales paroxísticas hipersincrónicas y desorganizadas'. De los 3.5 millones de pacientes con epilepsia en todo el mundo, el $40 \%$ son menores de 15 años. México presenta una prevalencia de 15-18 casos/1,000 habitantes, semejante al resto de Latinoamérica ${ }^{2,3}$. Existen pocos estudios acerca de la calidad de vida relacionada con la salud (CVRS) en estos niños ${ }^{4,5}$. Al evaluarla deben considerarse aspectos como: desarrollo neurocognitivo, grado de madurez y nivel de comprensión ${ }^{4-10}$.

La escala de calidad de vida para pacientes epilépticos pediátricos (ECAVIPEP), validada en México, evalúa pacientes de 4 a 10 años. Contiene 22 preguntas en cinco dominios: autopercepción, motor grueso, comportamiento, cognitivo y socialización, con resultados de mala, regular y buena CVRS. Se responde en menos de 10 minutos. Para niños de 4 a 7 años, un encuestador lee las preguntas. La ECAVIPEP demostró una consistencia interna por alfa de Cronbach de 0.673, y una validez convergente de 0.67 por rho de Spearman, con base en 114 pacientes. Para constructos subjetivos, las escalas de autorreporte (como ECAVIPEP) se consideran como estándar de criterio ${ }^{11}$.

También en México se validó la escala de sobrecarga del cuidador de Zarit, para cuidadores primarios de pacientes con enfermedades crónico-degenerativas. Consta de 22 ítems de tres factores: impacto del cuidado, interpersonal y expectativas de autoeficacia. Los resultados posibles son: ninguna o poca sobrecarga, sobrecarga leve a moderada, moderada a severa, y severa ${ }^{12-15}$.

Existen pocos estudios en Latinoamérica, por lo que el conocimiento de la CVRS en niños con epilepsia y la influencia de los cuidadores sobre ella es limitado. Conocerla es importante para comprender el proceso de la enfermedad y su impacto individual y social, también para identificar las condiciones actuales de los pacientes y sus familias y además, para optimizar la calidad y la eficacia de la atención mediante un abordaje multidisciplinario. El objetivo de este trabajo fue correlacionar la CVRS del niño con epilepsia con la sobrecarga de sus cuidadores en un hospital de segundo nivel de atención médica en Puebla, México, del Instituto Mexicano del Seguro Social (IMSS).

\section{Métodos}

El presente trabajo fue aprobado por el Comité Local de Investigación y Ética en Investigación en Salud n.. 2102 del IMSS (con registro R-2017-2102-19). Se realizó un estudio descriptivo, prospectivo, transversal, realizado en un hospital de segundo nivel del IMSS en Puebla, México. A los pacientes reclutados se les aplicó la ECAVIPEP, y al adulto cuidador primario acompañante, la escala de Zarit. Se incluyó a niños de 4 a 10 años de edad con epilepsia, que acudieron a la consulta externa de neurología pediátrica, con tratamiento de seis meses o más y de menos de cinco años. Se excluyó a pacientes con patologías concomitantes independientes de la epilepsia que per se limitaran su CVRS. Se eliminó a aquellos (o cuyos padres) que no completaron las escalas.

\section{Análisis estadístico}

Se utilizó estadística descriptiva, prueba de correlación de Spearman y $X^{2}$ en el programa SPSS para Mac de IBM. Se consideró estadísticamente significativa una $\mathrm{p}<0.05$.

\section{Resultados}

Se reclutó a 74 pacientes. Se eliminaron a dos pacientes que no completaron la aplicación de ECAVIPEP, y 
Tabla 1. Resultados sociodemográficos de los pacientes incluidos en el estudio $(n=71)$

\begin{tabular}{|c|c|c|}
\hline \multicolumn{3}{|c|}{ Género } \\
\hline Masculino & \multicolumn{2}{|r|}{ Femenino } \\
\hline $39(55 \%)$ & \multicolumn{2}{|r|}{$32(45 \%)$} \\
\hline \multicolumn{3}{|c|}{ Edad de inicio } \\
\hline Lactante & Preescolar & Escolar \\
\hline $24(34 \%)$ & $28(39 \%)$ & $19(27 \%)$ \\
\hline \multicolumn{3}{|c|}{ Entorno académico } \\
\hline Acuden a la escuela & \multicolumn{2}{|r|}{ No acuden } \\
\hline $67(94 \%)$ & \multicolumn{2}{|r|}{$4(6 \%)$} \\
\hline \multicolumn{3}{|c|}{ Rendimiento escolar } \\
\hline Bajo & Promedio & Encima del promedio \\
\hline $19(27 \%)$ & $50(70 \%)$ & $2(3 \%)$ \\
\hline \multicolumn{3}{|c|}{ Tipo de crisis } \\
\hline Generalizadas & \multicolumn{2}{|r|}{ Parciales } \\
\hline $57(80 \%)$ & \multicolumn{2}{|r|}{$14(20 \%)$} \\
\hline \multicolumn{3}{|c|}{ Control de la epilepsia } \\
\hline Controlada & \multicolumn{2}{|c|}{ Descontrolada } \\
\hline $34(48 \%)$ & \multicolumn{2}{|r|}{$37(52 \%)$} \\
\hline \multicolumn{3}{|c|}{ Terapia antiepiléptica } \\
\hline Monoterapia & \multicolumn{2}{|r|}{ Politerapia } \\
\hline $55(77 \%)$ & \multicolumn{2}{|r|}{$16(23 \%)$} \\
\hline
\end{tabular}

uno cuyo cuidador no contestó la escala de Zarit. En los 71 pacientes restantes hubo un predominio del género masculino. La edad promedio fue de 6.7 años y la edad de inicio de las crisis se registró mayoritariamente en edades de lactancia y preescolar (Tabla 1).

Casi todos nuestros pacientes acuden a la escuela. De los cuatro pacientes (5.63\%) sin matrícula escolar, tres $(4.22 \%)$ no acuden por descontrol de las crisis y uno $(1.4 \%)$ por problemas de horario laboral de la madre, estando los padres divorciados. El rendimiento escolar en nuestra población se mantiene en su mayoría en el rango promedio (Tabla 1).

La monoterapia antiepiléptica predominó, el 7.04\% $(n=5)$ pasó de monoterapia a politerapia (Tabla 1).

Predominaron epilepsias de crisis generalizadas sobre focales (ver variantes en la figura 1). Solo se detectó un síndrome epiléptico (síndrome de Doose), considerado una combinación de crisis generalizadas mioclónica, astática y de ausencia.
Tabla 2. Sobrecarga de los cuidadores primarios

\begin{tabular}{|l|c|c|c|c|c|c|}
\hline \multirow{2}{*}{ Sobrecarga } & \multicolumn{2}{|c|}{ Madres } & \multicolumn{2}{|c|}{ Abuelos } & \multicolumn{2}{c|}{ Totales } \\
\cline { 2 - 8 } & $\mathbf{n}$ & $\%$ & $\mathbf{n}$ & $\%$ & $\mathbf{n}$ & $\%$ \\
\hline Poca o leve & 33 & 46 & 5 & 7 & 38 & 53 \\
\hline Leve a moderada & 19 & 27 & 7 & 10 & 26 & 37 \\
\hline Moderada a intensa & 3 & 4 & 3 & 4 & 6 & 8 \\
\hline Intensa & 1 & 1 & 0 & 0.00 & 1 & 1 \\
\hline Totales & 56 & 79 & 15 & 21 & 71 & 100 \\
\hline
\end{tabular}

Al momento del estudio, la frecuencia de pacientes con epilepsia controlada, en su mayoría con un periodo de dos a tres años libre de crisis, prácticamente se equilibró con la descontrolada. De los casos de epilepsia descontrolada, aquellos con una crisis o más en un semestre fueron los más frecuentes, seguidos de los de una crisis o más al día, al bimestre y al año (Fig. 2).

Los cuidadores primarios fueron las madres y los abuelos. Hasta el $46.5 \%$ reportaron algún grado de sobrecarga, siendo los(as) abuelos(as) quienes manifestaron mayor esfuerzo y cansancio en el cuidado de los pacientes (Tabla 2).

En la escala de Zarit, el factor interpersonal resultó sin afectación en el $66.66 \%(n=47.33)$, el impacto del cuidado, en el $51.64 \%$ ( $n=35.67)$, y el factor de autoeficacia, en el $32.39 \%(n=23)$. Este último fue el más comprometido (67.61\%). El $70.42 \%(n=50)$ reportaron un núcleo familiar íntegro (madre y padre viviendo juntos).

La mayoría de los cuidadores primarios de nuestros pacientes se reportó sin sobrecarga o con sobrecarga leve, aun entre la epilepsia no controlada (Tabla 3).

Las correlaciones con significado estadístico fueron: sobrecarga de cuidadores y frecuencia de crisis epilépticas (Spearman $=0.338 ; p=0.001)$, y sobrecarga de cuidadores y núcleo familiar (Spearman $=-0.673 ; p$ $<0.001$ ).

La calidad de vida en el niño con epilepsia fue buena en el $76.05 \%(n=54)$ de los casos y regular en el $23.95 \%(n=17)$, y no hubo mala calidad de vida (Fig. 3).

De acuerdo con los resultados de la ECAVIPEP, Ios dominios evaluados se observaron sin alteraciones en orden decreciente: motor grueso $(69.30 \%)$, autopercepción (56.34\%), socialización (48.89\%), comportamiento $(45.77 \%)$ y cognitivo $(31.99 \%)$. 


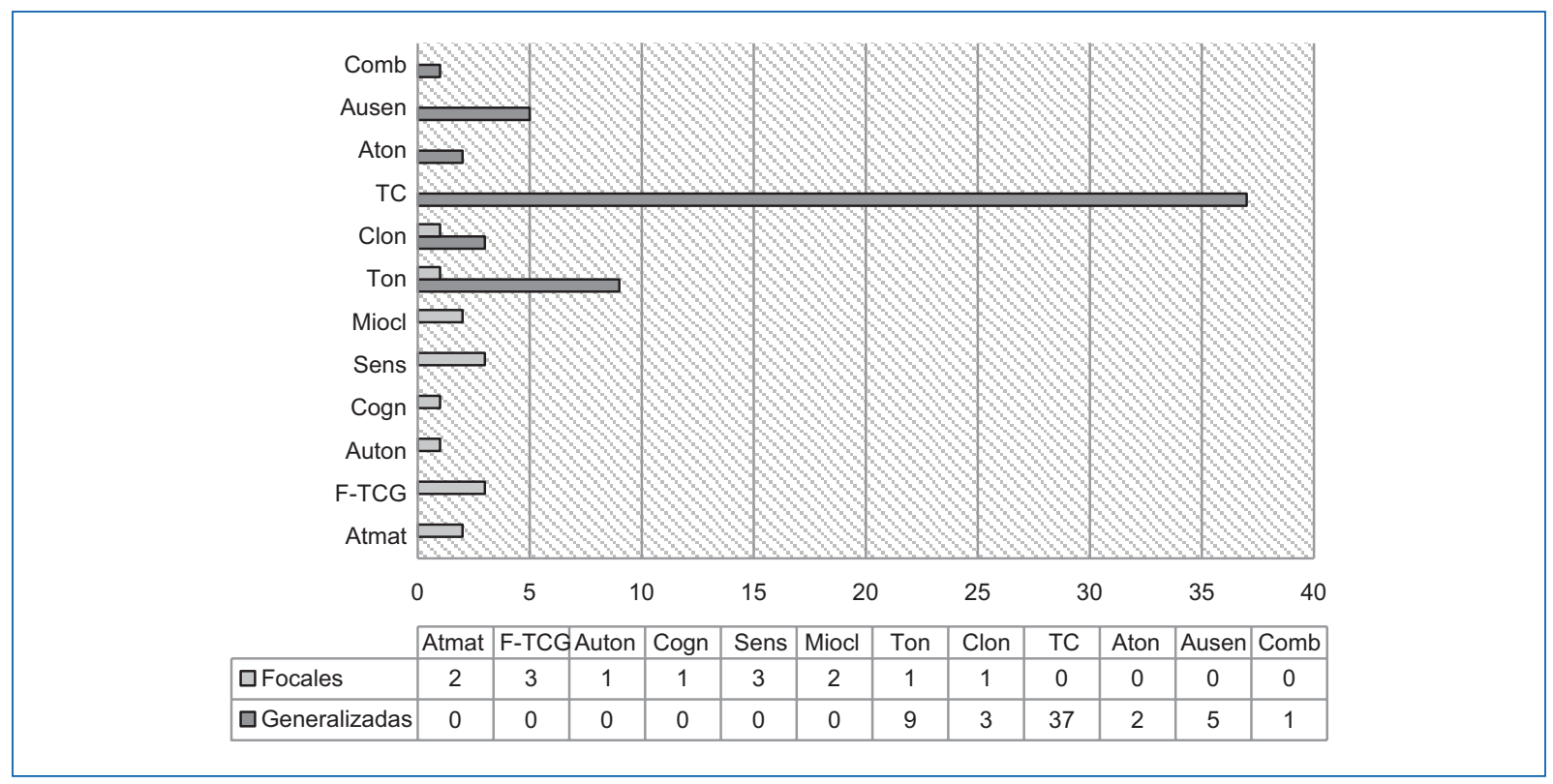

Figura 1. Tipos y frecuencias de crisis epilépticas. Atmat: automatismos; F-TCG: de focal a tónico-clónicas generalizadas; Auton: autonómicas; Cogn: cognitiva; Sens: sensitiva; Miocl: mioclónicas; Ton: tónicas; Clon: clónicas; TC: tónicoclónicas; Aton: atónicas; Ausen: ausencias; Comb: combinadas. (Elaboración propia, 2020).

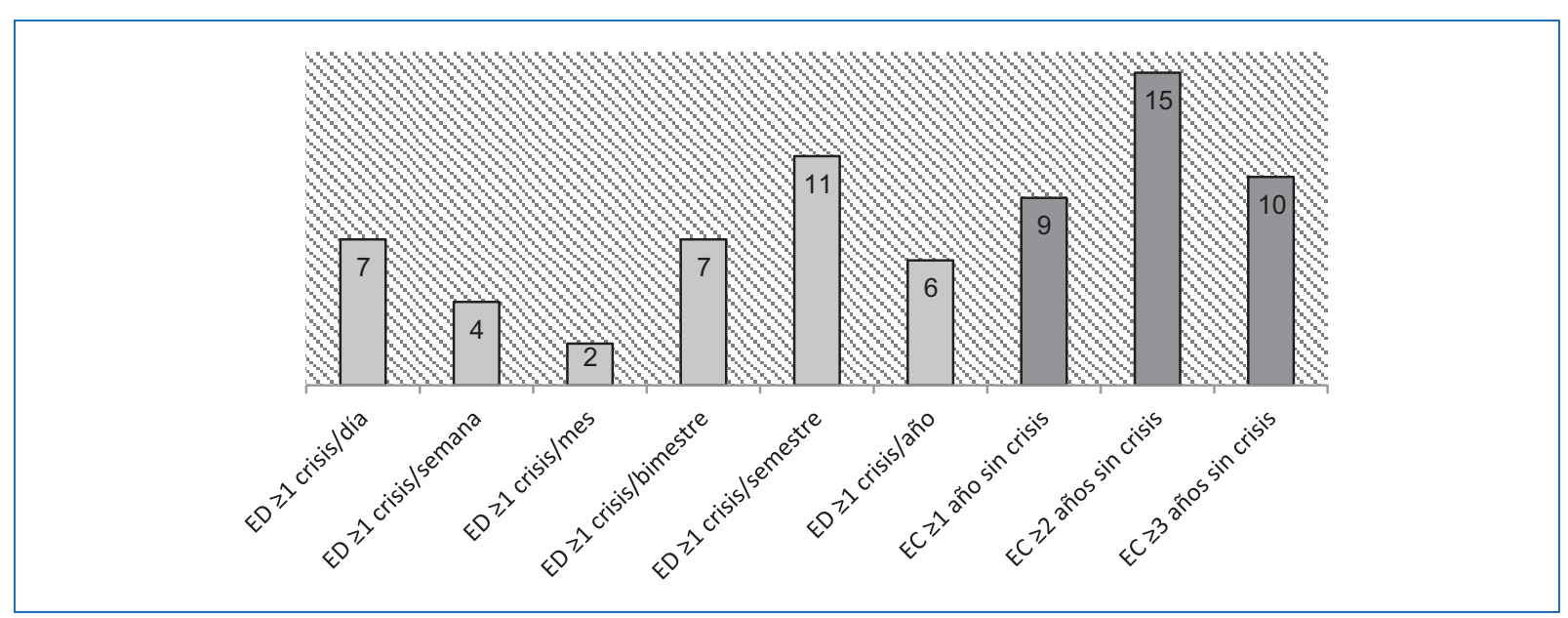

Figura 2. Control de la epilepsia. EC: epilepsia controlada; ED: epilepsia descontrolada.

La relación entre la frecuencia de las crisis y la CVRS obtuvo una Rho de Spearman $=-0.247$ ( $p=0.038$ ), y la relación entre sobrecarga de los cuidadores y CVRS del niño con epilepsia resultó con rho de Spearman $=-0.360$ $(p=0.002)$ y $X^{2}=0.083$ (Tabla 3).

\section{Discusión}

Nuestros resultados concuerdan en general con los de reportes internacionales en el predominio del género masculino (55\% en este trabajo versus $52.9-63.1 \%$ en otros reportes); la edad de inicio de 0 a 5 años (lactantes y preescolares); y en la mayor frecuencia de epilepsia generalizada y predominio de tónico-clónica (67.07\%) (Tabla 1 y Fig. 1) 3,8,11,16-20. Cabe mencionar que casos con afectación cognitiva mayor (como síndromes clásicos de West, Lennox-Gastaut, Dravet, etc.) se excluyeron del estudio, ya que no se les podía aplicar la ECAVIPEP.

En cuanto al control de la epilepsia y la farmacoterapia (mono o politerapia), nuestros datos también 
Tabla 3. Relación entre la calidad de vida, el control de la epilepsia y la sobrecarga de los cuidadores

\begin{tabular}{|l|c|c|c|c|}
\hline $\begin{array}{l}\text { Sobrecarga } \\
\text { del cuidador }\end{array}$ & \multicolumn{2}{|c|}{ Control de la epilepsia } & \multicolumn{2}{|c|}{$\begin{array}{c}\text { Calidad de vida } \\
\text { escala ECAVIPEP }\end{array}$} \\
\cline { 2 - 5 } & $\begin{array}{c}\text { Controlada } \\
(\%)\end{array}$ & $\begin{array}{c}\text { No } \\
\text { controlada } \\
(\%)\end{array}$ & $\begin{array}{c}\text { Buena } \\
(\%)\end{array}$ & $\begin{array}{c}\text { Regular } \\
(\%)\end{array}$ \\
\hline $\begin{array}{l}\text { No hay } \\
\text { sobrecarga }\end{array}$ & 38 & 18 & 48 & 6 \\
\hline $\begin{array}{l}\text { Sobrecarga } \\
\text { leve }\end{array}$ & 11 & 25 & 24 & 13 \\
\hline $\begin{array}{l}\text { Sobrecarga } \\
\text { moderada }\end{array}$ & 3 & 3 & 3 & 6 \\
\hline $\begin{array}{l}\text { Sobrecarga } \\
\text { intensa }\end{array}$ & 0 & 1 & 1 & 0 \\
\hline Totales & 52 & 47 & 76 & 25 \\
\hline
\end{tabular}

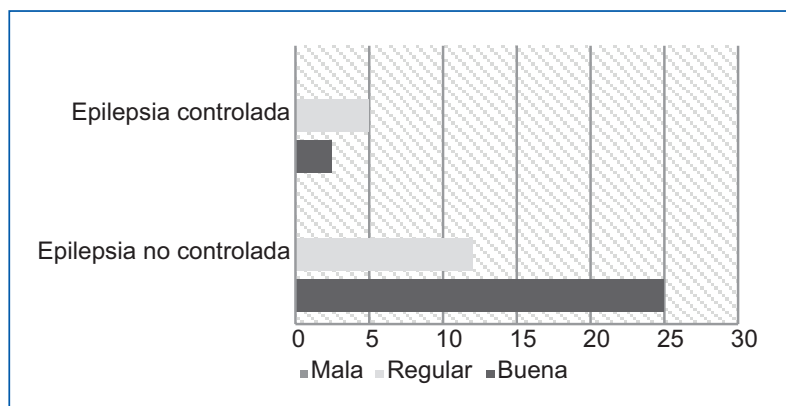

Figura 3. Calidad de vida y control de la epilepsia.

coinciden con otros estudios $8,13,17$ (Tabla 1). El porcentaje de epilepsia controlada (un año libre de crisis o más) resultó en el $47.88 \%$ (de ellos, el $38 \%$ con monoterapia); y el porcentaje de nuestros pacientes con monoterapia fue mucho mayor (77.5\%) (Tabla $1 \mathrm{y}$ Fig. 2). Destaca que ninguno de nuestros pacientes recibía tratamiento no farmacológico (dieta cetogénica, cirugía, etc.), ya que esos pacientes son tratados y controlados en el tercer nivel de atención.

La Organización Mundial de la Salud define la CVRS como la percepción de los individuos de su posición en la vida referente a sus metas, expectativas, normas e intereses, en el contexto cultural y el sistema de valores en los que viven. Está relacionado con el grado de satisfacción de sí mismos. Es decir, la CVRS intenta medir el impacto en la vida del paciente, su estado de salud y los tratamientos que recibe ${ }^{7-9,11,21}$. Al respecto, sobresale que, de nuestros pacientes, el $76 \%$ reportan buena calidad y el $24 \%$, regular. Si bien es similar a otros trabajos ${ }^{11,16-20}$, ningún paciente reportó mala calidad, quizá por ser pacientes atendidos ambulatoriamente, presumiblemente sin complicaciones mayores $\mathrm{y} / \mathrm{o}$ recientes.

En México, el grupo más vulnerable a la epilepsia son los niños. Entre otros factores involucrados destacan: la estigmatización que sufren, las repercusiones en su CVRS y en la de sus cuidadores, y el seguimiento del tratamiento. Las principales limitaciones a la CVRS en estos niños se ubican en la esfera psicosocial, que resulta más afectada, con disminución en la cognición, atraso y fracaso escolar. Las necesidades del niño con epilepsia repercuten en el cuidador primario, le condicionan sobrecarga y efectos negativos físicos y emocionales. También afectan el sistema familiar, provocando disfunciones en la coordinación y responsabilidad de la atención dentro de la familia y aislamiento social ${ }^{11-22}$.

Todo esto se ilustra con nuestros pacientes, quienes reportaron afectación considerable en los dominios de socialización, comportamiento y cognitivo, destacando los niños que no acuden a la escuela (Tabla 1).

La mayoría de nuestros pacientes acuden a la escuela, probablemente porque son familiares de trabajadores. La literatura reporta afectación cognitiva y/o académica en estos niños, como problemas de aprendizaje 0 de aprovechamiento escolar ${ }^{11,19-25}$. También reportes de Iberoamérica concuerdan en que el dominio cognitivo es el más afectado (desde el 54.6 al $92.9 \%)^{2,8,11,17-21,23,24}$. En nuestro estudio, el dominio cognitivo de la ECAVIPEP resultó sin afectación en el $32 \%$, pero su rendimiento escolar se reportó regular $(70 \%)$ e incluso alto (3\%). Quizá la epilepsia afecte la autopercepción del rendimiento cognitivo, y la afectación cognitivo-académica repercuta escolarmente después de la primaria. La influencia de la edad de inicio de la enfermedad no fue evaluada en este trabajo. Esto sugiere fuertemente explorar las características cognitivas de los pacientes con epilepsia.

Se le llama cuidador primario a la persona que asume una responsabilidad, por cuenta propia, de una persona afectada en su capacidad funcional, incluyendo cuidados, asistencia y tareas de apoyo ${ }^{25-27}$. Existe sobrecarga objetiva (tareas físicas del cuidado del paciente) y subjetiva (sentimientos y emociones que se generan como resultado de la primera). La interacción entre el estrés de los cuidadores y la respuesta ante ello puede condicionar una sensación de sobrecarga y efectos negativos en la salud, tanto emocional como física ${ }^{25-29}$.

En cuanto a los resultados, al aplicar la escala de Zarit destaca que el $74 \%$ de los cuidadores reportó no 
tener sobrecarga o sobrecarga leve, esto relacionado quizá a la CVRS. Sin embargo, la amplia variación de resultados (desde nula hasta severa sobrecarga) coincide con cuidadores de niños con otras patologías crónicas ${ }^{28-34}$. Desafortunadamente no existen estudios semejantes de sobrecarga del cuidador del niño con epilepsia. La correlación (si bien baja) entre frecuencia de crisis y sobrecarga de cuidadores confirmaría el supuesto de que cuando hay descontrol de la epilepsia existe más sobrecarga.

En nuestro estudio, la madre frecuentemente se asume como cuidador primario, semejante a otros estudios $^{35-37}$. El núcleo familiar (padre-madre) se reportó más frecuentemente como «íntegro» (70.4\%), proporción similar a la encontrada en otros estudios en México (73\%), sin embargo, aún en estos casos, el cuidado generalmente recae mayoritariamente en la madre ${ }^{37}$. Al respecto, se encontró una gran correlación inversa y significativa entre sobrecarga y el núcleo familiar íntegro (rho $=-0.673 ; p<0.001$ ). Según otros reportes, la sobrecarga del cuidador es mayor cuando el cuidador es proveedor económico $28,29,36-40$, lo que infiere que nuestras madres no son proveedoras económicas familiares, o no las principales. Nuestra cifra de sobrecarga en cuidadores abuelos (21\%) concuerda con estudios donde los cuidadores reportan mayor sobrecarga a mayor edad, probablemente por disminución de la capacidad física y aumento de la prevalencia de enfermedades crónico-degenerativas ${ }^{28,29,33}$. Además, factores como gravedad de la patología, ansiedad y estrés también afectan la sobrecarga ${ }^{28,29,34-40}$. La mayoría de nuestra muestra mostró sobrecarga nula o leve, y buena calidad de vida del paciente. No obstante, sobresale que 31 de los 37 pacientes con epilepsia no controlada reportaron sobrecarga nula o leve (Tabla 2).

$\mathrm{Al}$ establecer las correlaciones entre la ECAVIPEP y la escala de Zarit, solo se encontraron dos estadísticamente significativas:

1. La correlación entre frecuencia de crisis epilépticas y CVRS es débil (Spearman $=-0.247 ; p=0.038$ ), quizá porque a menor frecuencia de aparición de crisis mejora la CVRS ${ }^{36-38}$.

2. La correlación entre CVRS del niño con epilepsia y la sobrecarga de los cuidadores también resultó apenas de débil a moderada (Spearman de -0.360; $p=$ 0.002 ), probablemente influida por otros factores ambientales o personales para la sobrecarga. Dentro de estos factores se incluyen la situación familiar, como lo sugieren nuestros resultados, que mostraron mayor correlación entre la sobrecarga del cuidador y la dupla padre-madre íntegra, como ya se mencionó ${ }^{36-40}$.
No soslayamos que el presente trabajo excluye a pacientes cuyo déficit cognitivo impide la respuesta de la ECAVIPEP11. Ellos tendrían que evaluarse con una escala indirecta como la Escala de Calidad de Vida del Niño con Epilepsia (CAVE) $)^{4-5}$.

Se evidencia la necesidad de evaluar la CVRS rutinariamente, y una base más amplia de datos que la presente nos posibilitaría comparar la CVRS y la sobrecarga del cuidador entre diferentes síndromes epilépticos en nuestra población.

Podemos concluir que:

- La sobrecarga de los cuidadores de niños con epilepsia fue poca o nula en la mayoría de los casos. Esta es mayor si el cuidador es el abuelo(a) y menor si el núcleo familiar permanece íntegro.

- La CVRS de los niños con epilepsia es mayoritariamente buena, con mejoría al controlar las crisis epilépticas (aunque con una correlación débil).

- No se demostró correlación significativa entre la CVRS del niño con epilepsia y la sobrecarga del cuidador, si bien ambas variables fueron reportadas con evaluaciones positivas.

\section{Conflicto de intereses}

Los autores declaran no tener conflicto de intereses alguno.

\section{Financiamiento}

Los autores no recibieron patrocinio para llevar a cabo este artículo.

\section{Responsabilidades éticas}

Protección de personas y animales. Los autores declaran que para esta investigación no se han realizado experimentos en seres humanos ni en animales.

Confidencialidad de los datos. Los autores declaran que han seguido los protocolos de su centro de trabajo sobre la publicación de datos de pacientes.

Derecho a la privacidad y consentimiento informado. Los autores declaran que en este artículo no aparecen datos de pacientes.

\section{Bibliografía}

1. Yusta-Izquierdo A. Crisis convulsivas. Concepto, clasificación y etiología. Emergencias. 2005;17(1):S68-S73.

2. Reséndiz-Aparicio JC. Epidemiología de la epilepsia en América Latina. En: Velasco-Monroy AL. Epilepsia. Un punto de vista latinoamericano. México: Alfil; 2013. p. 1-10.

3. Serrano-Martín J. Aspectos epidemiológicos de la epilepsia en un Hospital General de segundo nivel. Plast \& Rest Neurol. 2004;3:39-43. 
4. Herranz-Fernández JL, Casas-Fernandez C. Escala de calidad de vida del niño con epilepsia (CAVE). Rev Neurol. 1996;24(125):28-30.

5. Casas-Fernández C. Experiencia con el cuestionario de calidad de vida en el niño con epilepsia (CAVE). Rev Neurol. 1997;25(139):415-21.

6. Urzúa A, Caqueo A. Calidad de vida: una revisión teórica del concepto. Terapia Psicológica. 2012;30(1):61-71.

7. WHO Quality of Life Assessment Group. (1996): ¿Qué calidad de vida? Grupo de la OMS sobre la calidad de vida. Foro mundial de la salud 1996;17(4):385-387. Disponible en: http://www.who.int/iris/handle/10665/55264. (Última consulta el 12 de diciembre de 2019).

8. Iglesias-Moré S, Fabelo-Rochel JR, Rojas-Velázquez Y, González-Pal S, Ramírez-Muñoz A. Calidad de vida en niños y adolescentes con epilepsia. Rev Cubana Enferm. 2012;28(2):99-111.

9. Grupo de trabajo sobre calidad de vida en la infancia. Calidad de vida relacionada con la salud en pediatría. Instrumentos utilizados para su evaluación. Arch Argent Pediatr. 2014;112:571-6.

10. Velarde-Jurado E, Ávila-Figueroa C. Métodos para evaluar calidad de vida. Salud Pública de México. 2002:44 (5):448-60.

11. García-Galicia A, García-Carrasco M, Montiel-Jarquin AJ, García-Cuautitla MA, Barragán-Hervella RG, Romero-Figueroa MS. Validez y consistencia de las escalas ECAVIPEP y CAVE para evaluar la calidad de vida en pacientes pediátricos con epilepsia. Rev Neurol. 2014;59(7):301-6.

12. Montero-Pardo $Y$, Jurado-Cárdenas $S$, Valencia-Cruz A, Méndez Venegas J, Mora Magaña I. Escala del cuidador de Zarit: evidencia de validez en México. Psicooncología. 2014:11(1):71-85.

13. Stagg B, Larner A. Zarit Burden Interview: pragmatic study in a dedicated cognitive function clinic. Prog Neurol Psychiatry. 2015;19(4):198-202.

14. Zarit S, Reever K, Bach-Peterson J. Relatives of impaired early: correlates of feelings of burden. Gerontologist. 1980;20(1):649-55.

15. Alpuche-Ramírez VJ, Ramos-Del-Río B, Rojas-Rusell M, Figueroa-López CG. Validez de la Entrevista de Carga Zarit en una muestra de cuidadores primarios informales. Psicología y Salud. 2008;18(2):237-45.

16. Sillanpaa M. Learning disability: occurrence and long-term consequences in Childhood onset epilepsy. Epilepsy Behav. 2004;5:937-44.

17. Sokka A, Olsen P, Kirjavainen J, Harju M, Keski-Nisula L, Räisänen S, et al. Etiology, syndrome diagnosis, and cognition in childhood-onset epilepsy: a population-based study. Epilepsia Open. 2017;2:76-83.

18. López-Rojas V, Barragán-Pérez E, Hernández-Hernández M, Watemberg N, Legido S. Calidad de Vida en pacientes adolescentes con epilepsia en México. Rev Med Hondur. 2010;78:179-82.

19. Tavera-Saldaña LM, Enrique-Álvarez A, Izquierdo-Bello A, Cabrera SC Vásquez P, Uscátegui OL. Caracterización de los problemas de aprendizaje en niños y adolescentes con epilepsia primaria generalizada en la liga central contra la epilepsia y el hospital de La Misericordia (Bogotá Colombia). Medicina. 2011;33:227-48

20. Alvarado-Tobias M, Barragán-Pérez E, Tercero-Quintanilla G. Valoración de la calidad de vida en epilepsia, mediante el uso de QOLCE-55, en pacientes atendidos en el Hospital Infantil de México Federico Gómez. Rev Mex Neuroci. 2016;17:S218-9.

21. Ramírez-Rodríguez SM. Diseño de una escala para evaluar Calidad de Vida en niños y adolescentes con epilepsia ECANIVAE-LICCE. Universidad Nacional de Colombia; 2015. En: bdigital.unal.edu.co. Disponible en: http://bdigital.unal.edu.co/50902/1/33817908.2015.pdf. (Última consulta el 12 de diciembre de 2018).

22. Chong L, Jamieson NJ, Gill D, Singh-Grewal D, Craig JC, Ju A, et al Children's experience of epilepsy: a systematic review of qualitative studies. Pediatrics. 2016;138(3):e20160658.
23. Dávila-Ávila M, Delgado-De-la-Mora J, Candia-Plata MC, Álvarez-Hernández G. Calidad de vida relacionada con la salud en niños con epilepsia. Rev Neurol. 2014;59(2):63-70.

24. Rodríguez-Blancas y Herrero MC. Calidad de vida en niños y adolescentes epilépticos mexicanos en consulta de neuropediatria: Escala de CAVE. Rev Mex Neuroci. 2014;15(1):18-22.

25. Rivas-Herrera J, Ostiguín-Meléndez R. Cuidador: ¿concepto operativo o preludio teórico? Rev Enf Univ ENEO-UNAM. 2011:8:49-54.

26. Ruíz-Ríos E, Nava-Galán M. Cuidadores: responsabilidades-obligaciones. Enf Neurol Mex. 2012;11:163-9.

27. Canché-May J, Góngora-Coronado E, Leos-Ostoa Y, et al. Estilos de enfrentamiento del cuidador primario en los pacientes del CRIT Yucatán. Rev Elec Psic Izt. 2015;18:1729-44.

28. Camacho-Estrada L, Yokebed-Hinostrosa-Arvizu G, Jiménez-Mendoza A Sobrecarga del cuidador primario de personas con deterioro cognitivo y su relación con el tiempo de cuidado. Rev Enf Univ ENEO-UNAM. 2010;7:35-40.

29. Thompson R, Kerr M, Glynn M, Linehan C. Caring for a member with intellectual disability and epilepsy: Practical, social and emotional perspectives. Seizure. 2014;23:856-63.

30. Mar J, Arrospide A, Begiristain JM, Larrañaga I, Sanz-Guinea A Quemada I. Calidad de vida y sobrecarga de los cuidadores de pacientes con daño cerebral adquirido. Rev Esp Geriatr Gerontol. 2011; 46(4):200-5.

31. Díaz-Benítez E, Rodríguez-Martínez L. Comparación de la calidad de vida y sobrecarga entre cuidadores primarios de pacientes cardiópatas en edad pediátrica y adulta. Rev Mex Enferm Cardiol. 2014;22(1):6-12.

32. Montero-Pardo X, Jurado-Cárdenas S, Méndez-Venegas J. Carga, ansiedad y depresión en cuidadores primarios informales de niños con cáncer. Psicología y Salud. 2014;24(1):45-53.

33. López-Márquez G. Relación entre sobrecarga, ansiedad y depresión con la calidad de vida de cuidadores de niños con discapacidad. Tecnociencia Chihuahua. 2014;8(2):78-90.

34. Wightman A, Zimmerman CT, Neul S, Lepere K, Cedars KL, Opel D. Caregiver experience in pediatric dialysis. Pediatrics. 2019;143(2): e20182102.

35. Alonso-Babarro A, Garrido-Barral A, Díaz-Ponce A, Casquero-Ruiz R, Riera-Pastor M. Perfil y sobrecarga de los cuidadores de pacientes con demencia incluidos en el programa ALOIS. Aten Primaria. 2004;33(2): 61-8.

36. Ramaglia G, Romeo A, Viri M, Lodi M, Sacchi S, Cioffi G. Impact of idiopathic epilepsy on mothers and fathers: strain, burden of care, worries and perception of vulnerability. Epilepsia. 2007;48(9):1810-3.

37. Gutiérrez-Moctezuma J, Villegas-Peña H, Solórzano-Gómez E, Hernández-Rodríguez V. El impacto social y educacional por la epilepsia, en niños derechohabientes de tres hospitales de seguridad social. Rev Mex Pediatr. 2000;67(3):111-6.

38. Cianchetti C, Messina P, Pupillo E, Crichiutti G, Baglietto MG, Vaggiotti $P$, et al. The perceived burden of epilepsy: impact on the quality of life of children and adolescents and their families. Seizure. 2015;24(3):93-101.

39. Timonet-Andreu E, Morales-Asencio J, Canca-Sánchez J. Calidad de vida relacionada con la salud y sobrecarga de cuidadores en pacientes ingresados con insuficiencia cardiaca. Enferm Cardiol. 2013;10(5859):50-5.

40. Macedo E, Silva L, Paiva M. Sobrecarga y calidad de vida en madres de niños y adolescentes con enfermedad crónica: una revisión integradora. Rev Latino-Am Enfermagem. 2015;23 (4):729-77. 